ARTICLE OPEN

\title{
Towards individualized diagnostics of biofilm-associated infections: a case study
}

Mathias Müsken ${ }^{1,2,5}$, Kathi Klimmek ${ }^{1,2}$, Annette Sauer-Heilborn ${ }^{3}$, Monique Donnert ${ }^{1,2}$, Ludwig Sedlacek ${ }^{4}$, Sebastian Suerbaum ${ }^{4,6}$ and $^{2}$ Susanne Häussler ${ }^{1,2}$

Organized within biofilm communities, bacteria exhibit resistance towards a broad spectrum of antibiotics. Thus, one might argue that bacteria isolated from biofilm-associated chronic infections should be subjected to resistance profiling under biofilm growth conditions. Various test systems have been developed to determine the biofilm-associated resistance; however, it is not clear to what extent the in vitro results reflect the situation in vivo, and whether the biofilm-resistance profile should guide clinicians in their treatment choice. To address this issue, we used confocal microscopy in combination with live/dead staining, and profiled biofilmassociated resistance of a large number $(>130)$ of clinical Pseudomonas aeruginosa isolates from overall 15 cystic fibrosis patients. Our results demonstrate that in addition to a general non-responsiveness of bacteria when grown under biofilm conditions, there is an isolate-specific and antibiotic-specific biofilm-resistance profile. This individual resistance profile is independent on the structural properties of the biofilms. Furthermore, biofilm resistance is not linked to the resistance profile under planktonic growth conditions, or a mucoid, or small colony morphology of the tested isolates. Instead, it seems that individual biofilm structures evolve during biofilm-associated growth and are shaped by environment-specific cues. In conclusion, our results demonstrate that biofilm resistance profiles are isolate specific and cannot be deduced from commonly studied phenotypes. Further clinical studies will have to show the added value of biofilm-resistance profiling. Individualized diagnosis of biofilm resistance might lead to more rational recommendations for antimicrobial therapy and, thus, increased effectiveness of the treatment of chronically infected patients.

npj Biofilms and Microbiomes (2017)3:22; doi:10.1038/s41522-017-0030-5

\section{INTRODUCTION}

Pseudomonas aeruginosa plays an important role in pulmonary infections of cystic fibrosis (CF) patients. ${ }^{1}$ Despite intensified antimicrobial therapy, chronic $P$. aeruginosa lung infection, repeated exacerbations, and progressive deterioration in lung function remain a major cause of morbidity and mortality. In the chronically infected CF lung $P$. aeruginosa adopts a biofilm mode of growth that provides a protected niche for the bacteria. ${ }^{2,3}$ Biofilm bacteria are much more resistant to antibiotic treatment, as well as to the host immune response, and it has been shown that with the formation of bacterial biofilms it becomes difficult, if not impossible, to eradicate the infection. ${ }^{4-8}$

Antimicrobial susceptibility testing is applied to guide clinicians in their treatment choices. However, antibiotic susceptibilities of planktonic populations as determined by conventional susceptibility test methods may not reflect the actual resistance profile of biofilm-associated infections. ${ }^{9}$ The use of minimal inhibitory concentration (MIC) test results for the treatment of clinical exacerbations that are thought to be caused mainly by planktonic bacteria is likely valid. However, it might be valuable to complement the MIC results with a test aimed to decide, which is the best antibiotic to be used as a maintenance therapy in order to suppress the chronic infection. ${ }^{3}$
Various methods have been developed to determine antibiotic resistance under biofilm growth conditions. ${ }^{10-13}$ There have also been several attempts to evaluate their predictive values as diagnostic tools in clinical trials. ${ }^{14-18}$ However, it still remains to be shown that susceptibility testing under biofilm growth conditions results in different recommendations for antimicrobial treatment as compared to MIC testing and that chronically infected CF patients indeed benefit from biofilm resistance profiling. ${ }^{19}$

One of the major needs for the evaluation of the value of biofilm resistance profiling for clinical outcome is the use of a standardized and reliable high-throughput system to monitor biofilm growth under the addition of various antibiotics. We have previously developed an optical method to provide information on the responsiveness of $P$. aeruginosa biofilms to increasing concentrations of various antimicrobial agents. ${ }^{20}$ BacLight viability staining in combination with automated confocal laser scanning microscopy (CLSM) on $P$. aeruginosa grown under biofilm conditions in a 96-well plate format proved to be a highly effective and rapid method to monitor the efficiency of various antibiotics. Application of the optical system also revealed information on the structure and constitution of the bacterial biofilm population.

In this study, we optimized the optical method for antibiotic susceptibility profiling of biofilm-grown $P$. aeruginosa and

\footnotetext{
${ }^{1}$ Institute for Molecular Bacteriology, TWINCORE, Centre for Experimental and Clinical Infection Research, Hannover, Germany; ${ }^{2}$ Department of Molecular Bacteriology, Helmholtz Centre for Infection Research, Braunschweig, Germany; ${ }^{3}$ Department of Pulmonary Medicine, Hannover Medical School, Hannover, Germany and ${ }^{4}$ Institute for Medical Microbiology and Hospital Epidemiology, Hannover Medical School, Hannover, Germany Correspondence: Mathias Müsken (Mathias.muesken@helmholtz-hzi.de)

${ }^{5}$ Present address: Central Facility for Microscopy, Helmholtz Centre for Infection Research, Braunschweig, Germany

${ }^{6}$ Present address: Max von Pettenkofer Institute, Medical Microbiology and Hospital Epidemiology, München, Germany
}

Received: 10 April 2017 Revised: 28 August 2017 Accepted: 29 August 2017

Published online: 28 September 2017 


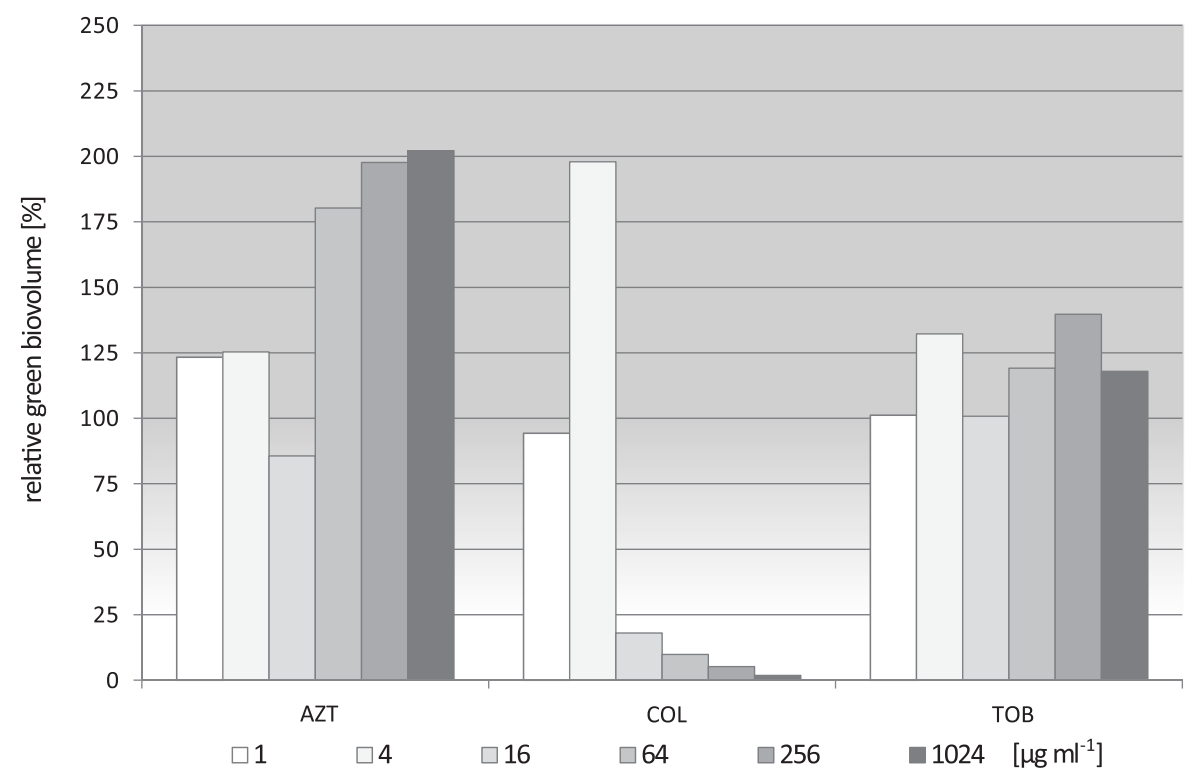

Fig. 1 Determination of the biofilm-active score (BAS) of the three antibiotics on $P$. aeruginosa biofilm-grown bacteria. An exemplary data set on the responsiveness of biofilm-grown bacteria of the clinical isolate Iso1525 towards various concentrations of the antibiotics aztreonam (AZT), colistin (COL), and tobramycin (TOB) is shown. We used the untreated control to set the corresponding green biovolume values as the $100 \%$ vitality value. The green biovolume can increase to values larger than $100 \%$, if biofilm formation is induced by non-lethal antibiotic concentrations (a common observation). The isolate Iso1525 is non-responsive towards the activity of AZT and TOB, but concentrations $\geq 16$ $\mu \mathrm{g} \mathrm{ml}^{-1} \mathrm{COL}$ lead to a reduction in the green biovolume by $>75 \%$. This corresponds to a BAS of +++ . The BAS is dependent on the green biovolume reduction and the minimal antibiotic concentration necessary to reach the reduction. For details of BAS categorizations see Table S2

determined biofilm-resistance profiles, as well as standard MIC and minimal bactericidal concentration (MBC) values of a large collection of clinical CF isolates. Most of the adult CF patients receive antibiotics for permanent inhalation therapy to suppress bacterial growth. Thus, we tested three antibiotics which are currently available for inhalation: aztreonam, colistin, and tobramycin. High concentrations of these antibiotics can be reached within the CF lung despite significant inter-patient variation. ${ }^{21-26}$

Our results demonstrate that there are isolate-specific and antibiotic-specific biofilm-resistance profiles and that the individual profiles cannot be deduced from other isolate-specific characteristics, such as the structure of the biofilm or the MIC values of the individual clinical isolates. An individualized diagnostics of biofilm-associated resistance might therefore overcome the limitations of conventional resistance testing for the prediction of treatment success/failure and thus might improve quality of health care measures in chronically infected patients.

\section{RESULTS}

Collection of clinical isolates and determination of the biofilmactive score (BAS)

Overall, $113 P$. aeruginosa isolates were collected from $15 \mathrm{CF}$ patients over a period of two and a half years (Table S1). An additional 20 isolates were sampled as a follow up for 13 out of 15 patients. On average, nine $P$. aeruginosa isolates were obtained from each patient with a minimum of five isolates and a maximum of 15 . Some of the patient's sputum samples contained a morphologically diverse population including small colony variants (SCVs) or mucoid isolates, others contained only one morphotype. The recovery of a morphological diverse population from chronically infected sites is well described and seems to reflect bacterial adaptation to hostile environments. ${ }^{27-29}$

We grew the 133 clinical isolates within biofilms in 96-well plates and subjected them to serial dilutions of the inhalative antibiotics aztreonam, colistin and tobramycin. Following a live and dead staining, those biofilms were analyzed using CLSM.
The green (live) biovolume was monitored and the relative reduction of the green biovolume upon antibiotic treatment was determined. As exemplified in Fig. 1, we categorized the biofilm as "fully responsive" (with a BAS of +++ ) if the green biovolume was reduced by $>75 \%$ within a certain antibiotic concentration range (please see Table S2 for details), as "responsive" if the biovolume was reduced in the range of $50-75 \%$ (BAS++), as "weakly responsive" in the range $25-50 \%$ (BAS+), and as "non-responsive"/resistant (R) if a reduction of the green biovolume of less than $25 \%$ was observed. As demonstrated previously, ${ }^{20}$ biofilmresistance profiling was very robust and a concentrationdependent reduction could be observed for both, the green biovolume and the colony forming unit (CFU) counts (Fig. S1).

Comparison of antibiotic effectiveness on planktonic and biofilmgrown bacteria

Examples of acquired biofilm images and resistance data of two clinical isolates (Iso1525 and Iso0052) are presented in Fig. 2. The isolate Iso1525 exhibited MIC/MBC values in the resistant range for aztreonam and tobramycin, whereas the MIC values for colistin were in the sensitive range. As might have been expected, the biofilm-grown bacteria of the isolate were non-responsive towards the activity of aztreonam and tobramycin, but responsive towards the activity of colistin at the given concentration range.

Isolate Iso0052 exhibited MIC values in the resistant range towards the activity of colistin and tobramycin, and values in the sensitive range towards the activity of aztreonam. However, all the three antibiotics were effective as we observed an overall reduction in the green biofilm biovolume.

In Table S3, a summary of MIC, MBC, and BAS values for the 133 clinical isolates of all 15 patients is shown. Additionally, colony morphology characteristics (SCV, mucoidity) are recorded. Many of the isolates were very sensitive and showed MIC and MBC values below the tested antibiotic concentration range. 1, 51, and 4 of the clinical isolates exhibited MIC and MBC values of $\leq 1 \mu \mathrm{g} \mathrm{ml}^{-1}$ towards aztreonam, colistin, and tobramycin, respectively. 

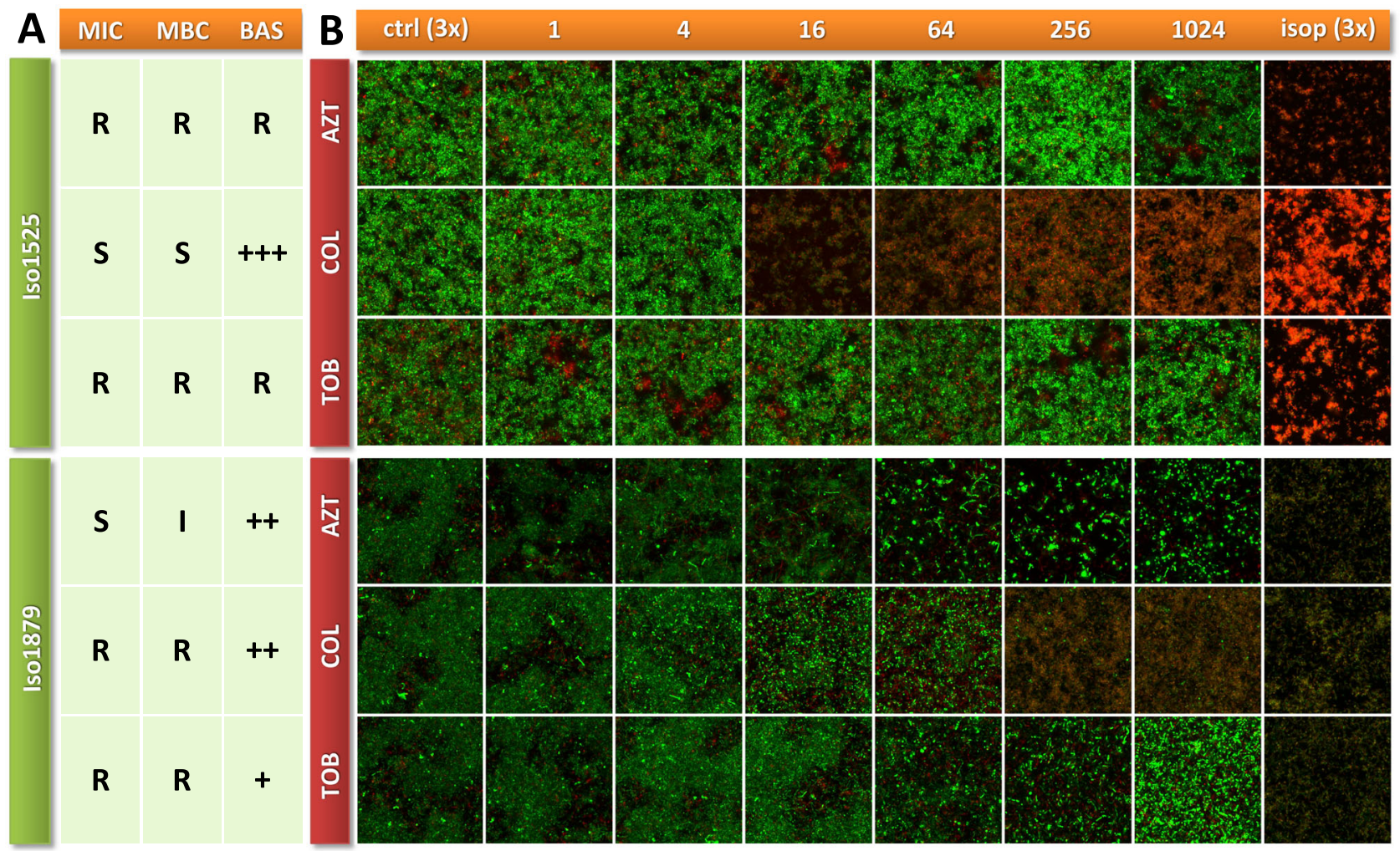

Fig. 2 Comparison of antibiotic resistance profiles. a Minimal inhibitory/bactericidal concentrations (MIC/MBC) and the biofilm-active score (BAS) of aztreonam (AZT), colistin (COL), and tobramycin (TOB) of the two clinical isolates Iso1525 and Iso0052. b Biofilm projections of single samples treated with increasing concentrations of antibiotic $\left[\mu \mathrm{g} \mathrm{ml}^{-1}\right]$. ctrl: non-treated control, isop: isopropanol control (triplicates). Fortyeight hours old biofilms are stained with the BacLight ${ }^{\mathrm{TM}}$ Viability-Kit, visualizing dead cells in red (propidium iodide) and living cells in green (Syto9)

Other isolates were highly resistant and exhibited MIC values above the tested concentration range of $\geq 128 \mu \mathrm{g} \mathrm{ml}^{-1}$ for aztreonam (43), colistin (5) and tobramycin (16 of the clinical isolates). For those isolates MBC values were not determined.

In general, the antibiotic concentration required to effectively kill the planktonically growing bacteria (MBC) was usually higher than the MIC ( 2 to $\geq 8$ times higher). Only in three clinical isolates the MIC and MBC were identical. A strongly increased $M B C$ as compared to the MIC (MBC/MIC: $\geq 8 \times$ ) was mainly found for aztreonam and colistin in a number of strains (42 of $89(47 \%)$ and 33 of $76(43 \%)$, respectively), this number was two times lower for tobramycin (24 of 113 (21\%) isolates).

Most of the aztreonam resistant (MIC) clinical isolates were also non-responsive towards the antimicrobial activity if grown under biofilm conditions (50 out of 62 isolates, Table 1). In addition, 21 out of 23 aztreonam intermediate resistant isolates were resistant towards the activity of aztreonam if grown under biofilm conditions. These results corborate previous findings of an increased resistance of isolates grown under biofilm conditions. However, 12 of the resistant (according to their MIC) and two of the intermediate resistant isolates were responsive towards aztreonam under biofilm growth conditions. Vice versa, from the overall 22 aztreonam-sensitive isolates (MIC values of $\leq 1 \mu \mathrm{g} \mathrm{ml}^{-1}$ ), 14 isolates (Table 1) were resistant towards the activity of aztreonam if grown under biofilm conditions and eight were responsive.

Similar results were obtained for tobramycin (Table 1). Twentyeight of the 40 clinical strains that exhibited MIC values in the resistant range were non-responsive towards tobramycin under biofilm-growth conditions, whereas 12 strains proved to be responsive (BAS of,+++ , or +++ ). Vice versa 49 of the 74 tobramycin-sensitive isolates were non-responsive towards the activity of tobramycin if cultured under biofilm conditions, whereas 25 were responsive.

Colistin seems to effectively kill the bacteria even under biofilmgrowth conditions (Table 1). $92 \%$ of the colistin resistant isolates (12 of 13 isolates) exhibited a BAS of at least ++ . On the other hand, only 1 of the 102 colistin-sensitive isolates exhibited a BAS in the non-responsive range. More than $53 \%$ (61 out of 115) of the clinical isolates were fully responsive $(B A S+++)$ and more than $29 \%$ (33 out of 115 ) were responsive (BAS++) towards the activity of colistin (total: 82\%) if grown under biofilm conditions. In contrast only $10 \%$ (11 out of 107) and 20\% (23 out of 114) of the clinical isolates exhibited a BAS of ++ or +++ towards the activity of aztreonam and tobramycin, respectively.

Our results thus demonstrate that resistance values increase if the bacteria are grown under biofilm conditions. However, some clinical strains exhibit an unexpected responsiveness or nonresponsiveness towards antibiotic activity under biofilm growth conditions.

Link between colony morphologies and planktonic and biofilmresistance patterns

To evaluate whether the colony morphologies impact on resistance profiles, we compared the planktonic and biofilmresistance profiles of the mucoid ( $n=25$ isolates from overall eight patients) and SCV ( $n=38,12$ patients) isolates to that of the whole community $(n=100,33$ isolates have been excluded due to missing BAS values).

As depicted in Fig. 3, the SCV phenotype was associated with higher MIC values, whereas the mucoid strains were more susceptible as compared to the overall population against all three of the tested antibiotics. This higher susceptibility of mucoid 
Table 1. Comparison of antibiotic resistance profiles

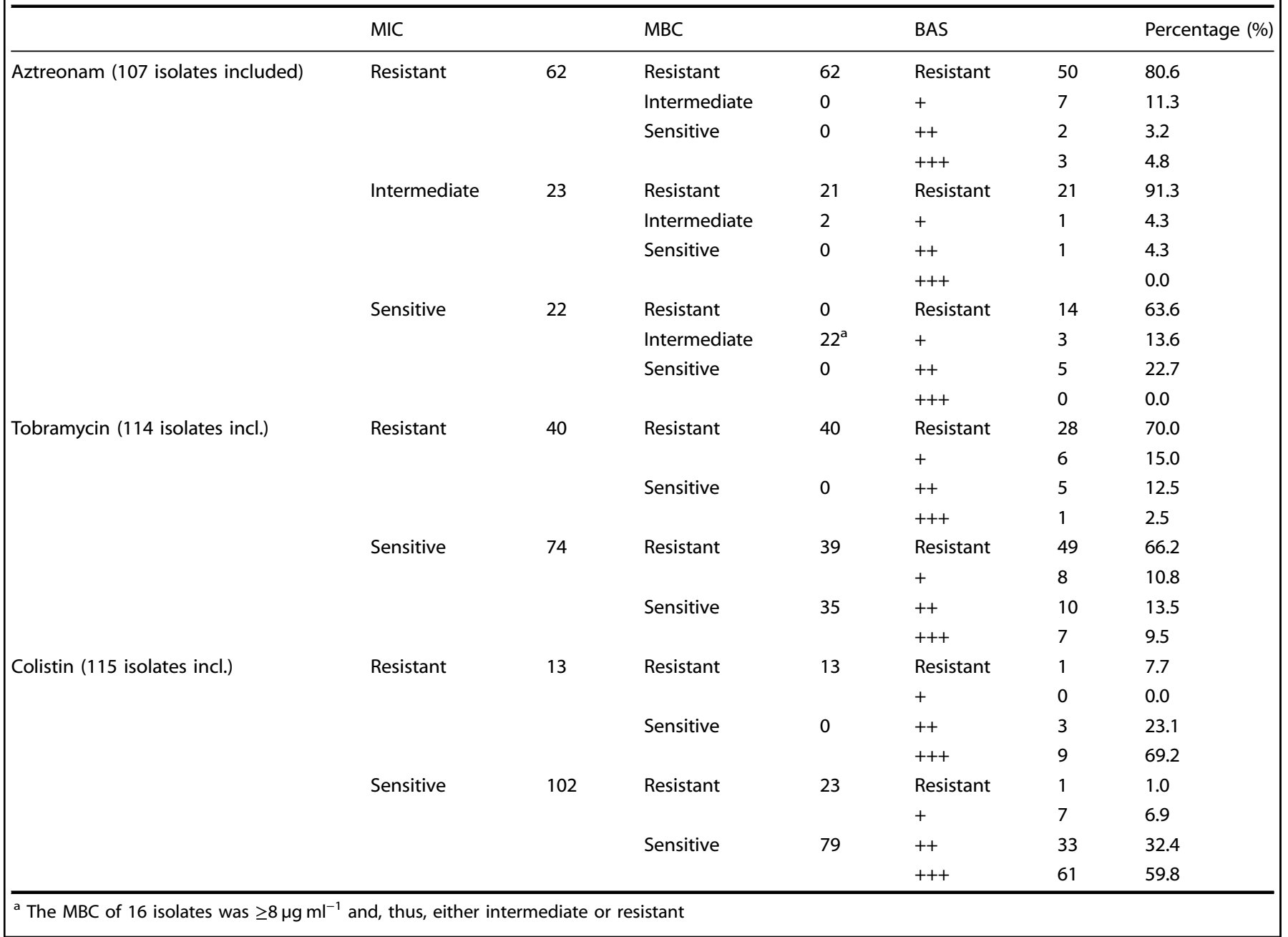

isolates has been described before. ${ }^{30,31}$ However, in contrast to the planktonic conditions, mucoid isolates seem to be as resistant or even slightly more antibiotic resistant as compared to the overall population if grown under biofilm conditions.

Correlation between the $P$. aeruginosa biofilm structure, colony morphology, and the biofilm-resistance profile

Within the collection of 133 isolates from the overall $15 \mathrm{CF}$ patients, not only different colony morphotypes were observed, but also morphologically distinct biofilm phenotypes as determined by CLSM (Fig. S2). Some of the biofilms were rather flat, whereas others exhibited distinctive structures. Of note, there did not seem to be a correlation between colony morphology (SCV, mucoid and other) and the biofilm phenotype. The various mucoid or SCV isolates did not exhibit specific biofilm structures compared to the other clinical isolates (Fig. 4). Furthermore, we did not observe a correlation of the biofilm structure and the biofilm-active scores (Fig. 5).

$P$. aeruginosa biofilm structure across different CF patients over time

In general, as expected, biofilm phenotypes of the infecting $P$. aeruginosa isolates exhibited various biofilm phenotypes across the different CF patients (high inter-patient variability). Furthermore, in some patients the various isolates that were recovered from one sputum sample exhibited quite distinct biofilm phenotypes (intra-patient variability) (Figs. S2 and S3). Nevertheless similar biofilm phenotypes also appeared recurrently in different patients, indicating that there are biofilm phenotypes (as they become apparent in the in vitro system) that are formed again and again (Fig. S4).

Interestingly, in most of the patients the biofilm phenotypes were stable and could be repeatedly observed in subsequently isolated P. aeruginosa strains (e.g. patients 11, 12, and 14, Fig. S2). Furthermore, isolates from the same patients tended to form similar biofilm structures even if they expressed different colony morphotypes (patients 7 and 12, Fig. S3). This indicates that the patient-specific microenvironment seems to shape the biofilm phenotype and that this microenvironment can be found repeatedly in the lungs of various CF patients.

\section{DISCUSSION}

We have recently established a method, which allows susceptibility testing of biofilm-grown $P$. aeruginosa isolates. In this study, the method was adjusted to susceptibility testing of the inhaled antimicrobial agents aztreonam, colistin, and tobramycin. Susceptibility profiles of 133 biofilm-grown clinical $P$. aeruginosa isolates, recovered from the respiratory tract material of overall $15 \mathrm{CF}$ patients were determined. We aimed to address the question of whether an individual testing of the resistance profile under biofilm-growth conditions might be justified. Biofilm-resistance 
Total

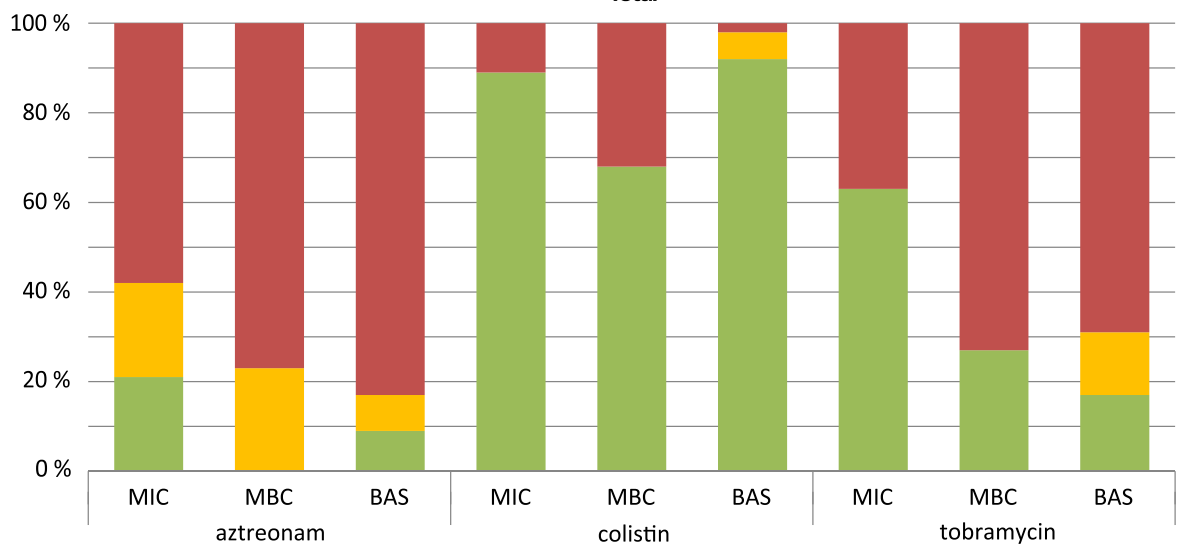

SCV morphotype

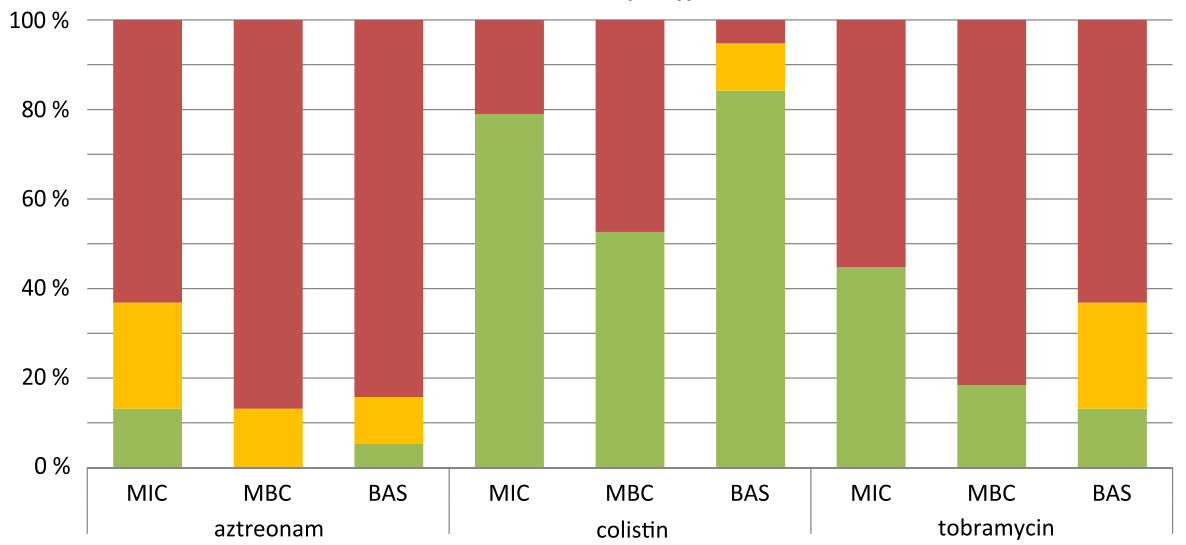

Mucoid morphotype

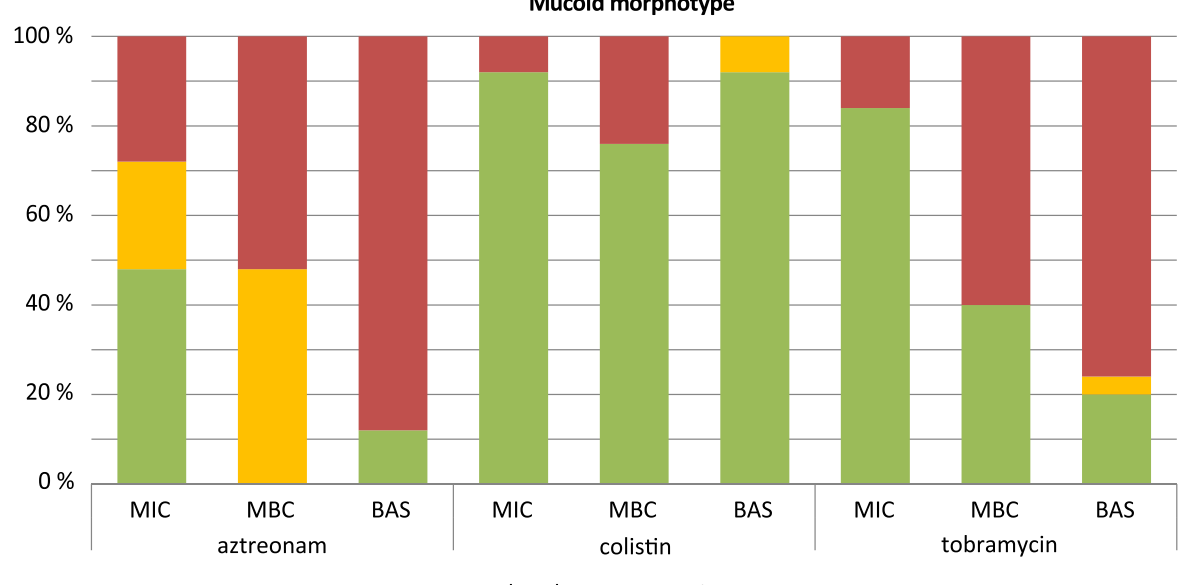

$\square \mathrm{S} /++/+++\square \mathrm{I} /+\quad \square \mathrm{R}$

Fig. 3 Planktonic but not biofilm-resistance profiles differed between morphotypes. The distribution of susceptible (S), intermediate (I), and resistant $(\mathrm{R})$ isolates towards the respective antibiotic are shown. The BAS categories ++ and +++ were defined as susceptible and category + as intermediate. In a data of all isolates $(n=100)$ is shown, in $\mathbf{b}$ and $\mathbf{c}$ the data of SCVs $(n=38)$ and the mucoid morphotype $(n=25)$ are shown, respectively. Both morphotypes did not overlap since there was no mucoid SCV

profiling might be worth the effort, if biofilm-resistance profiles cannot be inferred from the MIC values.

A plethora of studies have previously demonstrated that biofilm growth protects the bacteria from the activity of a many different antimicrobials. . $^{52-37}$ In agreement with this, we observed that the inhalative antibiotics were generally less effective under biofilmgrowth conditions. However, we also observed strain-dependent differences in the effectiveness of the inhalative antibiotics under biofilm-growth conditions and those differences were independent of the MIC profiles. The discordance of MIC and BAS profiles was most apparent for aztreonam and tobramycin. $19.4 \%$ of the aztreonam and $30.0 \%$ of the tobramycin resistant (according to the MIC) isolates were still responsive to the antimicrobials under biofilm-growth conditions, whereas 63.6 and $66.2 \%$ of the sensitive (according to the MIC) isolates were nonresponsive under biofilm-growth conditions. Of note, whereas many clinical isolates were almost completely non-responsive towards the activity of aztreonam and tobramycin under 


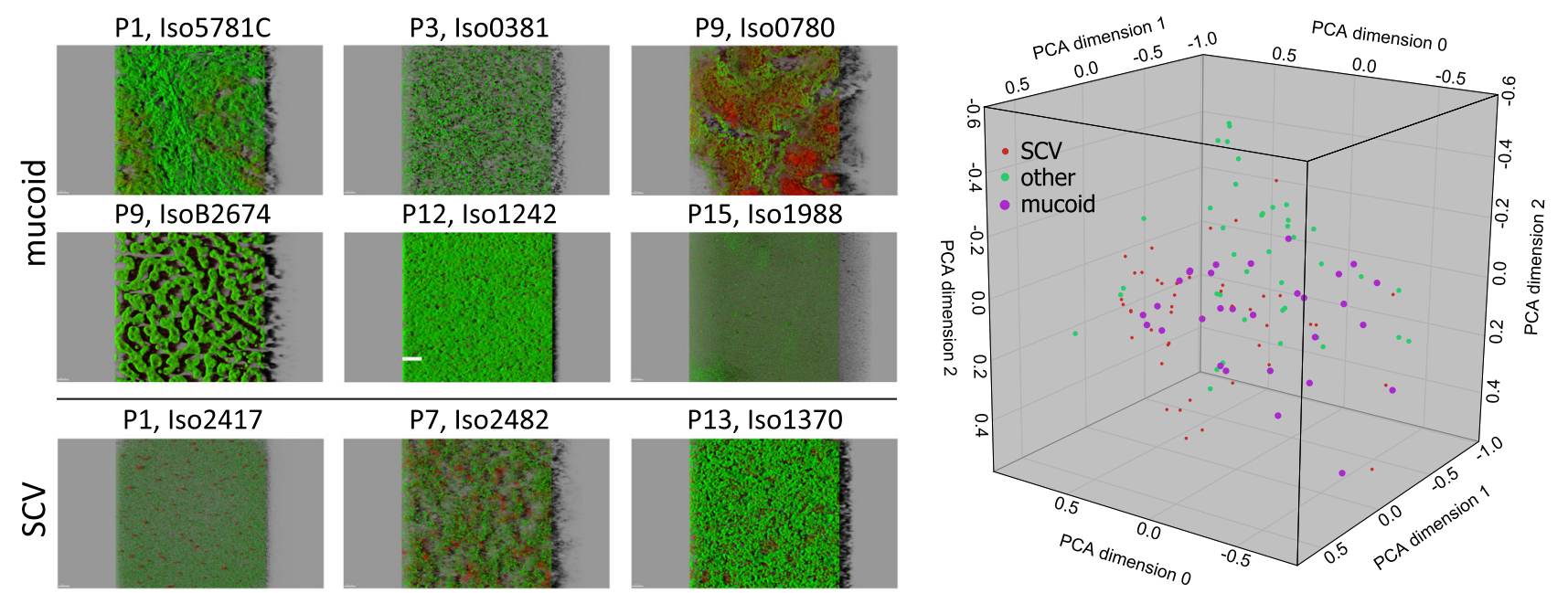

Fig. 4 Mucoid or SCV clinical isolates do not form specific biofilm structures. a Examples of biofilm phenotypes of selected mucoid and SCV morphotypes visualized in an easy-3D projection ( $48 \mathrm{~h}$-old biofilms stained with the BacLight ${ }^{\mathrm{TM}}$ Viability-Kit) and $\mathbf{b}$ principal component analysis (PCA) representation of the biofilm phenotype of 113 clinical isolates. Seven biofilm parameters were taken into account. SCV are depicted in red, mucoid in pink, and others in green
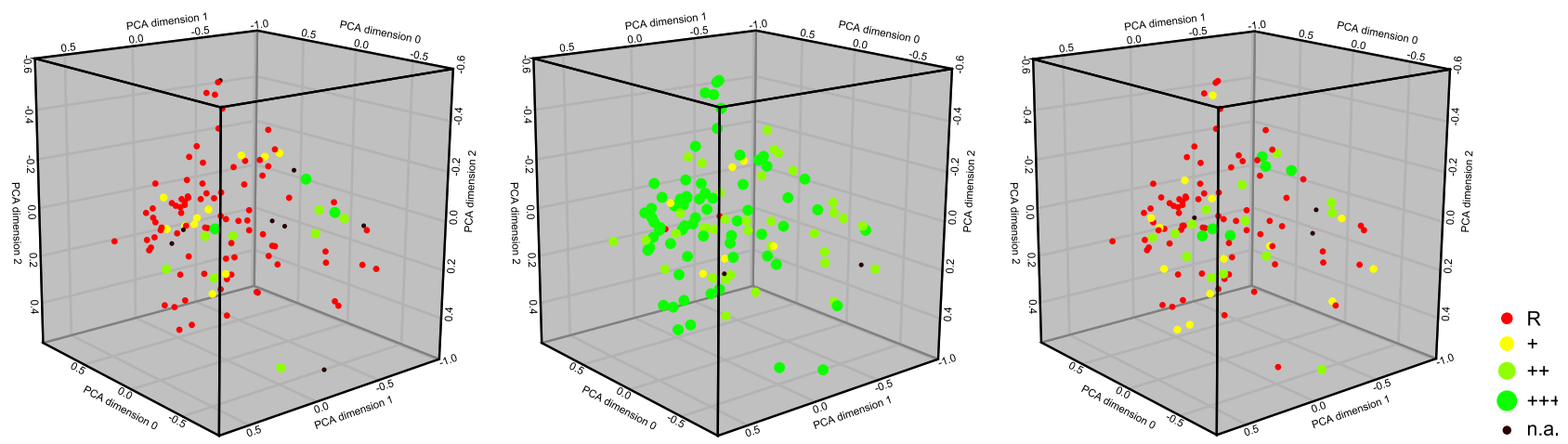

Fig. 5 Expression of distinct biofilm structures do not correlate with particular biofilm-active scores. PCA representation of the biofilm phenotype of 113 clinical isolates. The BAS categories for $\mathbf{a}$ aztreonam, $\mathbf{b}$ colistin, and $\mathbf{c}$ tobramycin are depicted. The colors represent resistant (red) isolates and isolates with a BAS category of + (yellow), ++ (light green), and +++ (green)

biofilm-growth conditions, colistin seemed to be fully active. The bactericidal activity of colistin against $P$. aeruginosa biofilms has been shown to be enhanced under anaerobic/microaerophilic conditions, ${ }^{38}$ which are also found in microtiter plate assays, ${ }^{39,40}$ as well as in CF lungs. ${ }^{41}$

Our results clearly demonstrate that in order to increase antibiotic effectiveness in chronic biofilm-associated infections, only individual testing of the biofilm-resistance profile will give the full information on which antibiotic could be most effective. This holds particularly true for the inhalative antibiotics, aztreonam and tobramycin, and seems to be less relevant for colistin.

Interestingly, testing of the biofilm responsiveness towards the activity of the three antibiotics revealed no clear cross-resistance. This indicates that mechanisms of biofilm resistance are, in addition to a general tolerance of biofilm-grown bacteria towards external stresses at least in parts due to strain-specific characteristics, to develop resistance against single antibiotics. In this study, we also observed that the colony morphologies impacted the MIC profiles. Mucoid isolates were generally more sensitive and SCVs more resistant to either of the antibiotics tested in this study. This phenomenon has been described before. ${ }^{30,42-46}$ In contrast, colony morphologies did not impact the biofilm structure, nor did they influence the responsiveness of biofilm-grown bacteria towards the activity of the inhalative antibiotics.

This underscores our finding that biofilm resistance is indeed independent on the MIC profile and that the individual infecting
$P$. aeruginosa isolates do exhibit distinct biofilm responsiveness that is intrinsically and stably linked to the bacterial genotype.

In conclusion, in this pilot study we have carefully evaluated biofilm susceptibility profiles of a collection of clinical isolates. Our finding of clearly discordant results between planktonic and biofilm resistances implies that individual testing of the resistance profiles under biofilm-growth conditions might be worth the effort in those clinical isolates, which have been recovered from a chronic biofilm-associated infected site. Biofilm-resistance profiling as a novel diagnostic measure might significantly impact on a more rational and more effective antimicrobial inhalative therapy of chronically $P$. aeruginosa infected CF patients. Nevertheless, large clinical trials will be required in order to demonstrate that biofilm-resistance profiles indeed lead to a more reliable positive predictive value for clinical success/failure of inhaled antimicrobial therapy.

\section{METHODS}

\section{Bacterial cultivation}

Clinical $P$. aeruginosa isolates were isolated from respiratory tract samples at the Institute of Medical Microbiology and Hospital Epidemiology at the Hannover Medical School and collected over a period of 2.5 years (2010-2012) from 15 patients. In total, 113 isolates could be recovered. Additional 20 isolates from 13 patients (no additional isolates from patients 6 and 7) have been collected $\sim 3$ years after the initial study period. Isolates 
were routinely cultivated on Columbia agar plates with $5 \%$ sheep blood (Becton Dickinson) to determine the colony morphotype (mucoid and SCV). Glycerol stocks were stored at $-80^{\circ} \mathrm{C}$ until use.

\section{Determination of the MIC and MBC}

For MIC determinations, single colonies of overnight grown isolates were suspended in $0.9 \% \mathrm{NaCl}$. Optical densities $\left(\mathrm{OD}_{600}\right)$ were adjusted to 0.01 and mixed with serially diluted antibiotics in $\mathrm{LB}$ medium within a microtiter plate to a final cell concentration in the well of $5 \times 10^{6} \mathrm{cells} \mathrm{ml}^{-1}$. The antibiotic test ranges of aztreonam, colistin, and tobramycin were $1,2,4,8$, 16,32 , and $64 \mathrm{\mu g} \mathrm{ml}^{-1}$. After overnight cultivation $(\sim 16 \mathrm{~h})$, the microtiter plates were visually inspected and the MIC (the lowest antibiotic concentration that prevented visible growth) was recorded. Clinical breakpoints were defined according to the European Committee on Antimicrobial Susceptibility Testing (EUCAST) classification to categorize susceptible (S) and resistant (R) isolates, including intermediate resistant (I) isolates for aztreonam.

For MBC determinations, we spotted $100 \mu$ (total volume) of the MICplate wells without visible growth (MIC and two higher concentrations) into single wells of a 24-well plates filled with LB agar (1.5\%). Growth was evaluated after a minimum of $24 \mathrm{~h}$ of incubation (plates were kept longer to allow evaluation of slow growing isolates, e.g. SCVs). The lowest antibiotic concentration without visible growth was recorded as the MBC. All experiments were performed in triplicates.

\section{Determination of biofilm resistance}

Biofilm susceptibility testing was performed as previously described. ${ }^{20}$ Briefly, bacterial pre-cultures were grown in $\mathrm{LB}$ and diluted to an $\mathrm{OD}_{600}$ of 0.002. Biofilm formation in LB medium accommodated growth of the majority of clinical isolates and proved to be stable and robust. Hundred microliters per well was used as inoculum for biofilm growth in a $\mu$ clear half area plate (Greiner Bio-one). Bacteria were allowed to establish a biofilm for $24 \mathrm{~h}$ at $37^{\circ} \mathrm{C}$ in a humid atmosphere. Bacterial biofilms were then exposed to antibiotics at the following concentrations: 1, 4, 16, 64, 256 , and $1024 \mathrm{\mu g} \mathrm{ml}^{-1}$. The addition of distilled water and isopropanol served as a growth and killing control, respectively. In parallel, dyes of the BacLight Viability kit (Molecular Probes, Inc.) were added to differentiate live (Syto9) and dead (propidium iodide) bacteria. After another growth period of $24 \mathrm{~h}$, bacteria were analyzed via confocal laser-scanning microscopy (CLSM). In one 96-well plate, resistance profiles of two isolates were tested against three antibiotics (Fig. S5).

To determine CFUs, dilution series (1:10) of resuspended biofilms were spotted onto agar plates using a 96-pin replicator.

\section{Confocal microscopy}

Automated microscopy was performed with an inverted SP8 system (Leica Microsystems) and the Leica application suite LAS X including the Matrix screener tool. To obtain biofilm images, two image stacks were acquired in parallel at the center of a well. Both stacks were acquired with a 40x/NA 1.1 water objective and the same laser and detector settings. However, stack 1 (overview job) was acquired using a zoom $\times 0.75$ (image size: $\sim 387 \times 387 \mu \mathrm{m}$, pixel size of $0.378 \mu \mathrm{m}$ ) and a total range of $\sim 80 \mu \mathrm{m}$ (27 slides and a slice distance of $3 \mu \mathrm{m}$ ), while stack 2 (zoom job) was acquired with a zoom $\times 4$ (image size: $\sim 73 \mu \mathrm{m} \times 73 \mu \mathrm{m}$; pixel size of 0.142 $\mu \mathrm{m})$ to visualize single cells. The stack size of the zoom job was reduced to $30 \mu \mathrm{m}$ ( 10 slides $\times 3 \mu \mathrm{m}$ ). Both stacks started at position zero which is the substratum (foil) found by a reflection-based autofocus run. For the automated image acquisition of all samples, a number of pre-defined laser/ detector settings were assigned to compensate inter-species and inter-well fluctuations in intensity avoiding under-exposed and over-exposed images.

\section{Image-analysis and visualization}

Both acquired image stacks were analyzed with the Developer XD (Definiens) software. The programmed customized solution of the Developer software is based on a previously described software called $\mathrm{PHLIP}^{47}$ and is used to determine biofilm-specific parameter for both fluorescent channels separately, as well as combined including, for example, ratios of the differentially stained populations within the biofilm or the biovolume, which describes the biofilm biomass. To optimize the analysis, we combined data of the overview and the zoom image stacks; the biovolume was taken from the overview job to define the overall amount of biofilm, while we used the percentage of dead (red fluorescent) cells of the zoom job. The latter has a better image resolution and, thus, allowed a more accurate estimation of fluorescence ratios. With both values we determined the green fluorescent biofilm - the living population -and compared treated samples with the control. A series of antibiotic dilutions are needed to categorize the responsiveness. ImageJ and Imaris (Bitplane AG) were used for the visualization of biofilm image stacks, the latter for complex $3 \mathrm{D}$ reconstructions.

\section{Principal component analysis (PCA)}

Seven biofilm parameters (biovolume $\left[\mu \mathrm{m}^{3}\right]$, area to volume $\left[\mu \mathrm{m}^{-1}\right]$, horizontal and vertical spreading $\left[\mu \mathrm{m}^{2}\right]$, mean thickness $[\mu \mathrm{m}]$, roughness $[-]$, and substrate coverage bottom [\%]) of a combined fluorescence channel (including fluorescence of green and red signals) were used for PCA using the software KNIME (version 3.3.1 ${ }^{48}$ ). In addition to the untreated controls (three samples), samples treated with $1 \mu \mathrm{g} \mathrm{ml}^{-1}$ antibiotic (three samples) have been included to determine more robust mean values of the biofilm parameters for each isolate, since the biofilm phenotype was not influenced by low antibiotic concentration (see Figs. 1 and 2). In total, 20 isolates have been excluded from PCA analysis due to missing image data in half of the replicas (weak green fluorescence in controls; contaminants in region of interest) or erroneous data analysis (BAS determination failed in minimum two of three antibiotics).

\section{Data availability}

The data that support the findings of this study are available from the corresponding author upon reasonable request.

\section{ACKNOWLEDGEMENTS}

This work was supported by the German Mukoviszidose e.V. [grant number 1401]. We thank Tanja Trompisch, Lidija Böshans, and Norina Molzen from the Institute for Medical Microbiology and Hospital Epidemiology (Hannover Medical School) for isolation of clinical $P$. aeruginosa isolates from respiratory tract samples.

\section{AUTHOR CONTRIBUTIONS}

S.H., A.S.-H., and M.M. planned the project. M.M., K.K., M.D., A.S.-H., and L.S. performed the experiments. S.S. and L.S. provided reagents and tools and M.M. and S.H. wrote the manuscript.

\section{ADDITIONAL INFORMATION}

Supplementary Information accompanies the paper on the npj Biofilms and Microbiomes website (https://doi.org/10.1038/s41522-017-0030-5).

Competing interest: Dr. Häussler and Dr. Müsken have a patent EP2459739 (Methods and Tests for Screening Bacterial Biofilms) licensed, and a patent US2012122729 issued.

Publisher's note: Springer Nature remains neutral with regard to jurisdictional claims in published maps and institutional affiliations.

\section{REFERENCES}

1. Tümmler, B. \& Kiewitz, C. Cystic fibrosis: an inherited susceptibility to bacterial respiratory infections. Mol. Med.Today 5, 351-358 (1999)

2. Bjarnsholt, T. et al. Pseudomonas aeruginosa biofilms in the respiratory tract of cystic fibrosis patients. Pediatr. Pulmonol. 44, 547-558 (2009).

3. Høiby, N. et al. ESCMID* guideline for the diagnosis and treatment of biofilm infections 2014. Clin. Microbiol. Infect. 21, S1-S25 (2015).

4. Parsek, M. R. \& Singh, P. K. Bacterial biofilms: an emerging link to disease pathogenesis. Annu. Rev. Microbiol. 57, 677-701 (2003).

5. Moskowitz, S. M., Foster, J. M., Emerson, J. \& Burns, J. L. Clinically feasible biofilm susceptibility assay for isolates of Pseudomonas aeruginosa from patients with cystic fibrosis. J. Clin. Microbiol. 42, 1915-1922 (2004).

6. Nickel, J. C., Ruseska, I., Wright, J. B. \& Costerton, J. W. Tobramycin resistance of Pseudomonas aeruginosa cells growing as a biofilm on urinary catheter material. Antimicrob. Agents Chemother. 27, 619-624 (1985).

7. Hoyle, B. D. \& Costerton, J. W. Bacterial resistance to antibiotics: the role of biofilms. Prog. Drug Res. 37, 91-105 (1991).

8. Bals, R., Hubert, D. \& Tümmler, B. Antibiotic treatment of CF lung disease: from bench to bedside. J. Cyst. Fibros. 10, S146-S151 (2011) 
9. Smith, A. L., Fiel, S. B., Mayer-Hamblett, N., Ramsey, B. \& Burns, J. L. Susceptibility testing of Pseudomonas aeruginosa isolates and clinical response to parenteral antibiotic administration: lack of association in cystic fibrosis. Chest 123, 1495-1502 (2003).

10. Chavant, P., Gaillard-Martinie, B., Talon, R., Hébraud, M. \& Bernardi, T. A new device for rapid evaluation of biofilm formation potential by bacteria. J. Microbiol. Methods 68, 605-612 (2007).

11. Pantanella, F. et al. BioTimer assay, a new method for counting Staphylococcus spp. in biofilm without sample manipulation applied to evaluate antibiotic susceptibility of biofilm. J. Microbiol. Methods 75, 478-484 (2008).

12. Ceri, H. et al. The calgary biofilm device: new technology for rapid determination of antibiotic susceptibilities of bacterial biofilms the calgary biofilm device: new technology for rapid determination of antibiotic susceptibilities of bacterial biofilms. J. Clin. Microbiol. 37, 1771 (1999).

13. Olivares, E. et al. The Biofilm Ring Test ${ }^{\oplus}$ : a rapid method for the routine analysis of P. aeruginosa biofilm formation kinetics. J. Clin. Microbiol. 54, 657-661 (2015).

14. Moskowitz, S. M. et al. Randomized trial of biofilm testing to select antibiotics for cystic fibrosis airway infection. Pediatr. Pulmonol. 46, 184-192 (2011).

15. Yau, Y. C. W. et al. Randomized controlled trial of biofilm antimicrobial susceptibility testing in cystic fibrosis patients. J. Cyst. Fibros. 14, 262-266 (2015).

16. Keays, T. et al. A retrospective analysis of biofilm antibiotic susceptibility testing: a better predictor of clinical response in cystic fibrosis exacerbations. J. Cyst. Fibros. 8, 122-127 (2009).

17. Hill, D. et al. Antibiotic susceptibilities of Pseudomonas aeruginosa isolates derived from patients with cystic fibrosis under aerobic, anaerobic, and biofilm conditions. J. Clin. Microbiol. 43, 5085-5090 (2005).

18. Aaron, S. D. et al. Combination antibiotic susceptibility testing to treat exacerbations of cystic fibrosis associated with multiresistant bacteria: a randomised, double-blind, controlled clinical trial. Lancet 366, 463-471 (2005).

19. Waters, V. \& Ratjen, F. Standard versus biofilm antimicrobial susceptibility testing to guide antibiotic therapy in cystic fibrosis. Cochrane Database Syst. Rev. CD009528. https://doi.org/10.1002/14651858.CD009528.pub3 (2015).

20. Müsken, M., Di Fiore, S., Römling, U. \& Häussler, S. A 96-well-plate-based optical method for the quantitative and qualitative evaluation of Pseudomonas aeruginosa biofilm formation and its application to susceptibility testing. Nat. Protoc. 5, 1460-1469 (2010).

21. Dalhoff, A. Pharmacokinetics and pharmacodynamics of aerosolized antibacterial agents in chronically infected cystic fibrosis patients. Clin. Microbiol. Rev. 27, 753-782 (2014).

22. Schuster, A., Haliburn, C., Döring, G. \& Goldman, M. H. Safety, efficacy and convenience of colistimethate sodium dry powder for inhalation (Colobreathe DPI) in patients with cystic fibrosis: a randomised study. Thorax 68, 344-350 (2013).

23. Yapa, S. W. S. et al. Pulmonary and systemic pharmacokinetics of inhaled and intravenous colistin methanesulfonate in cystic fibrosis patients: targeting advantage of inhalational administration. Antimicrob. Agents Chemother. 58, 2570-2579 (2014).

24. Pesaturo, K. A., Horton, E. R. \& Belliveau, P. Inhaled aztreonam lysine for cystic fibrosis pulmonary disease-related outcomes [English;Spanish] Aztreonam lisina inhalada y los resultados en la enfermedad pulmonar relacionada con fibrosis quistica. Ann. Pharmacother. 46, 1076-1085 (2012).

25. Sands, D., Sapiejka, E., Gaszczyk, G. \& Mazurek, H. Comparison of two tobramycin nebuliser solutions: pharmacokinetic, efficacy and safety profiles of T100 and TNS. J. Cyst. Fibros. 13, 653-660 (2014).

26. Gibson, R. L. et al. Microbiology, safety, and pharmacokinetics of aztreonam lysinate for inhalation in patients with cystic fibrosis. Pediatr. Pulmonol. 41, 656-665 (2006).

27. Burns, J. L. et al. Microbiology of sputum from patients at cystic fibrosis centers in the United States. Clin. Infect. Dis. 27, 158-163 (1998).

28. Foweraker, J. E., Laughton, C. R., Brown, D. F. J. \& Bilton, D. Phenotypic variability of Pseudomonas aeruginosa in sputa from patients with acute infective exacerbation of cystic fibrosis and its impact on the validity of antimicrobial susceptibility testing. J. Antimicrob. Chemother. 55, 921-927 (2005).

29. Clark, S. T. et al. Phenotypic diversity within a Pseudomonas aeruginosa population infecting an adult with cystic fibrosis. Sci. Rep. 5, 10932 (2015).

30. Ciofu, O., Fussing, V., Bagge, N., Koch, C. \& Høiby, N. Characterization of paired mucoid/non-mucoid Pseudomonas aeruginosa isolates from Danish cystic fibrosis patients: antibiotic resistance, beta-lactamase activity and RiboPrinting. $J$. Antimicrob. Chemother. 48, 391-396 (2001).
31. Häußler, S., Tümmler, B., Weissbrodt, H., Rohde, M. \& Steinmetz, I. Small-colony variants of Pseudomonas aeruginosa in cystic fibrosis. Clin. Infect. Dis. 29, 621-625 (1999).

32. Brooun, A., Liu, S. \& Lewis, K. A dose-response study of antibiotic resistance in Pseudomonas aeruginosa biofilms. Antimicrob. Agents Chemother. 44, 640-646 (2000).

33. Chiang, W. -C. et al. Extracellular DNA shields against aminoglycosides in Pseudomonas aeruginosa biofilms. Antimicrob. Agents Chemother. 57, 2352-2361 (2013).

34. De Kievit, T. R. et al. Multidrug efflux pumps: expression patterns and contribution to antibiotic resistance in Pseudomonas aeruginosa biofilms. Antimicrob. Agents Chemother. 45, 1761-1770 (2001).

35. Høiby, N., Bjarnsholt, T., Givskov, M., Molin, S. \& Ciofu, O. Antibiotic resistance of bacterial biofilms. Int. J. Antimicrob. Agents 35, 322-332 (2010).

36. Mah, T. -F. et al. A genetic basis for Pseudomonas aeruginosa biofilm antibiotic resistance. Nature 426, 306-310 (2003).

37. Mulcahy, H., Charron-Mazenod, L. \& Lewenza, S. Extracellular DNA chelates cations and induces antibiotic resistance in pseudomonas aeruginosa biofilms. PLoS Pathog. 4, e1000213 (2008).

38. Kolpen, M. et al. Increased bactericidal activity of colistin on Pseudomonas aeruginosa biofilms in anaerobic conditions. Pathog. Dis. 74, ftv086 (2016).

39. Cotter, J. J., O'Gara, J. P. \& Casey, E. Rapid depletion of dissolved oxygen in 96-well microtiter plate Staphylococcus epidermidis biofilm assays promotes biofilm development and is influenced by inoculum cell concentration. Biotechnol. Bioeng. 103, 1042-1047 (2009).

40. Diaz De Rienzo, M. A., Stevenson, P. S., Marchant, R. \& Banat, I. M. Effect of biosurfactants on Pseudomonas aeruginosa and Staphylococcus aureus biofilms in a BioFlux channel. Appl. Microbiol. Biotechnol. 100, 5773-5779 (2016).

41. Hassett, D. J. et al. Anaerobic metabolism and quorum sensing by Pseudomonas aeruginosa biofilms in chronically infected cystic fibrosis airways: rethinking antibiotic treatment strategies and drug targets. Adv. Drug Deliv. Rev. 54, 1425-1443 (2002).

42. Owlia, P., Nosrati, R., Alaghehbandan, R. \& Lari, A. R. Antimicrobial susceptibility differences among mucoid and non-mucoid Pseudomonas aeruginosa isolates. GMS Hyg. Infect. Control 9, Doc13 (2014).

43. Srifuengfung, S., Tiensasitorn, C., Yungyuen, T. \& Dhiraputra, C. Prevalence and antimicrobial susceptibility of Pseudomonas aeruginosa mucoid and non-mucoid type. Southeast Asian J. Trop. Med. Public Health 35, 893-896 (2004).

44. Drenkard, E. \& Ausubel, F. M. Pseudomonas biofilm formation and antibiotic resistance are linked to phenotypic variation. Nature 416, 740-743 (2002).

45. Morelli, P. et al. Characterization of_Staphylococcus aureus_small colony variant strains isolated from Italian patients attending a regional cystic fibrosis care centre. New Microbiol. 38, 235-243 (2015).

46. Kirisits, M. J., Prost, L., Starkey, M. \& Parsek, M. R. Characterization of colony morphology variants isolated from Pseudomonas aeruginosa biofilms. Appl. Environ. Microbiol. 71, 4809-4821 (2005).

47. Mueller, L. N., de Brouwer, J. F. C., Almeida, J. S., Stal, L. J. \& Xavier, J. B. Analysis of a marine phototrophic biofilm by confocal laser scanning microscopy using the new image quantification software PHLIP. BMC Ecol. 6, 1 (2006).

48. Berthold, M. R. et al. Knime. Web 1-8. https://doi.org/10.1007/978-3-540-78246-9 (2007).

Open Access This article is licensed under a Creative Commons Attribution 4.0 International License, which permits use, sharing, adaptation, distribution and reproduction in any medium or format, as long as you give appropriate credit to the original author(s) and the source, provide a link to the Creative Commons license, and indicate if changes were made. The images or other third party material in this article are included in the article's Creative Commons license, unless indicated otherwise in a credit line to the material. If material is not included in the article's Creative Commons license and your intended use is not permitted by statutory regulation or exceeds the permitted use, you will need to obtain permission directly from the copyright holder. To view a copy of this license, visit http://creativecommons. org/licenses/by/4.0/.

(c) The Author(s) 2017 